\title{
Mapping Molecular Orbital Symmetry on High-Order Harmonic Generation Spectrum Using Two-Color Laser Fields
}

\author{
Hiromichi Niikura, ${ }^{1,2}$ Nirit Dudovich, ${ }^{3}$ D. M. Villeneuve, ${ }^{1}$ and P. B. Corkum ${ }^{1,4}$ \\ ${ }^{1}$ National Research Council of Canada, 100 Sussex Drive, Ottawa, Ontario, Canada K1A0R6 \\ ${ }^{2}$ PRESTO, Japan Science and Technology Agency, Sanbancho Building, 5-Sanbancho, Chiyodaku, Tokyo, Japan, 102-0075 \\ ${ }^{3}$ Department of Physics of Complex Systems, Weizmann Institute of Science, Rehovot 76100, Israel \\ ${ }^{4}$ Department of Physics, University of Ottawa, 150 Louis Pasteur, Ottawa, Ontario, Canada K1N 6N5
}

(Received 7 June 2009; published 28 July 2010)

\begin{abstract}
We have measured high-order harmonic generation spectra of $\mathrm{D}_{2}, \mathrm{~N}_{2}$, and $\mathrm{CO}_{2}$ by mixing orthogonally polarized 800 and $400 \mathrm{~nm}$ laser fields. The intensity of the high-harmonic spectrum is modulated as we change the relative phase of the two pulses. For randomly orientated molecules, the phase of the intensity modulation depends on the symmetry of the molecular orbitals from which the high harmonics are emitted. This allows us to identify the symmetry of any orbital that contributes to high-harmonic generation, even without aligning the molecule. Our approach can be a route to imaging dynamical changes in three-dimensional molecular orbitals on a time scale as short as a few hundred attoseconds.
\end{abstract}

A high-harmonic generation spectrum contains information on atoms or molecules, such as vibrational motion [1,2], electron motion [3], and electronic structure [4-7]. Combined with molecular alignment techniques, molecular orbital tomography was demonstrated in 2004 [4], allowing us to measure the amplitude of an electronic wave function. However, there are major impediments to using molecular alignment methods for orbital tomography. First, there is an interplay between two basic processes underlying high-harmonic generation-tunnel ionization and electron recollision [8,9]. As we rotate the molecule for orbital tomography, tunneling probability is modulated and the structure of the recolliding electron changes $[10,11]$. Thus, the simplicity of the recolliding electron wave packet, on which orbital tomography is based, is lost. Second, low-lying orbitals can dominate at some angles for tunnel ionization probability $[12,13]$. It further complicates the high-harmonic generation process [14]. Third, only a relatively small class of molecules can be well aligned. These issues prohibit us from reconstructing molecular orbital with $\pi_{g}$ symmetry from the highharmonic spectra.

Another route for orbital tomography has been proposed by Shafir et al. [15]. They control electron trajectory in the continuum by applying an orthogonally polarized laser field with a wavelength of $400 \mathrm{~nm}$ in addition to the fundamental field at $800 \mathrm{~nm}$. This allows the atomic orbital symmetry to be clearly written in the high-harmonic spectrum.

We demonstrate how molecular orbital symmetry is probed using high-harmonic generation for $\mathrm{D}_{2}, \mathrm{~N}_{2}$, and $\mathrm{CO}_{2}$ by extending this approach. We use randomly aligned molecules, where harmonics are preferentially generated from a portion of ensembles which dominates the ionization probability. This minimizes the multiorbital effect on high-harmonic spectrum. For instance, for $\mathrm{CO}_{2}$ the ionization probability of the $\pi_{g}$ orbital is peaked at $45^{\circ}$ and $135^{\circ}$ from the molecular axis [16]. For a laser intensity of $1.9 \times$ $10^{14} \mathrm{~W} / \mathrm{cm}^{2}$, the probability at this angle is calculated to $1.1 \times 10^{14} / \mathrm{s}[13,16]$. This is two or three orders larger than those of the lower-lying $\pi_{u}$ and $\sigma_{g}$ orbitals at the molecular angles where the ionization probability has a maximum value. Thus, the $\pi_{g}$ orbital is most likely selected by ionization.

Figure 1 illustrates the ionization and recollision process schematically. We set the polarization axis of the $800 \mathrm{~nm}$ (or $400 \mathrm{~nm}$ ) laser field to the $x$ (or $y$ ) axis, respectively. We focus only on the angles in the plane perpendicular to the propagation direction of the driving laser pulses. This is because only the high-harmonic emission propagating along the driving laser fields can be well phase matched.

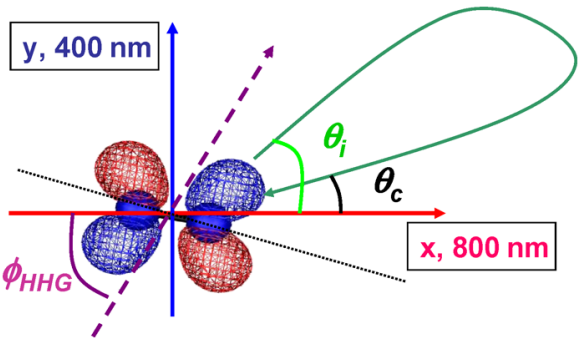

FIG. 1 (color online). A schematic picture of a molecular orbital and high-harmonic generation process. We set the polarization axis of an $800 \mathrm{~nm}(400 \mathrm{~nm})$ laser pulse to the $x$ axis ( $y$ axis), respectively. The combined fields detach an electron in the instantaneous maximum field direction at the angle of $\theta_{i}$, which determines the molecular alignment. The electron returns to the parent ion with the angle of $\theta_{c}$, leading to high-harmonic emission. For instance, at 13 th harmonic and at a relative delay of $\sim 0.6 \mathrm{fs}, \theta_{i}$ is $\sim 40$ and $\theta_{c}$ is $\sim 0^{\circ}$, respectively. The resulting high-harmonic emission for the $\pi_{g}$ orbital is polarized at $\phi_{\mathrm{HHG}} \sim 80^{\circ}$. Changing the relative delay between the two laser pulses changes $\theta_{i}, \theta_{c}$, and $\phi_{\mathrm{HHG}}$. Measuring $\phi_{\mathrm{HHG}}$ as a function of $\theta_{c}$ allows us to identify the orbital symmetry. 
The physics behind our measurement can be appreciated from the strong field approximation [17]. Under this approximation, an electron is preferentially ejected to the continuum to the direction of the vector sum of the two laser polarizations at the time of ionization. We refer to this angle as the ionization angle, $\theta_{i}$. For the $\pi_{g}$ orbital in $\mathrm{CO}_{2}$, the angular distribution of the ionization probability is peaked at $45^{\circ}$ with a mean width of $\sim 5^{\circ}$ [10]. It restricts those molecules which are responsible for high-harmonic generation to lie within the narrow cone centered along $\theta_{i} \pm 45^{\circ}$. After ionization, the electron is accelerated by the laser fields then recollides with the molecular ion at a certain angle, referred as to the recollision angle, $\theta_{c}$, leading to high-harmonic generation. The high-harmonic emission is polarized with the angle of $\phi_{\mathrm{HHG}}$. The polarization depends on both $\theta_{c}$ and the symmetry of the bound state wave function which recombines with the continuum electron wave packet. Thus, measurement of the relationship between $\phi_{\mathrm{HHG}}$ as a function of $\theta_{c}$ determines the symmetry of the bound state wave function. All angles are defined in the laboratory frame.

Instead of controlling molecular alignment, we change the phase delay between the two laser pulses, thereby changing the time-dependent field direction of the combined two-color laser fields. Changing the phase delay controls both $\theta_{c}$ and $\theta_{i}$. This approach has advantages over the previous molecular alignment approach [16]. First, the structure of the continuum electron wave packet is less dependent of the molecular alignment angle. Second, the ionized wave packet has a definite sign relative to the bound electron wave function. These features permit us to measure the relationship between $\phi_{\mathrm{HHG}}$ and $\theta_{c}$ more accurately for a $\pi_{g}$ orbital than the alignment approach, especially when $\theta_{c}$ is parallel to the molecular axis. Next, selecting the orientation with tunnel ionization allows us to probe molecules that cannot be easily aligned.

We measure the high-harmonic generation spectrum as a function of the relative phase delay between the two fields. Then at each delay, we obtain $\phi_{\mathrm{HHG}}$ through the highharmonic spectrum in the laboratory frame. Because of symmetry in the combined electric fields, both even and odd harmonics appear in the spectrum. The intensity of the odd (even) harmonics is proportional to the component parallel to the polarization direction of the $800 \mathrm{~nm}$ (400 nm) driving laser field [15]. Then the $\phi_{\mathrm{HHG}}$ is given by

$$
\phi_{\mathrm{HHG}}=\arctan \left(\sqrt{I_{2 \omega} / I_{\omega}}\right),
$$

where $I_{2 \omega}$ and $I_{\omega}$ are the intensities of the adjacent even and odd harmonic orders, respectively. In order to obtain $\phi_{\mathrm{HHG}}$ as a function of $\theta_{c}$ for each molecule, we use $\mathrm{D}_{2}$ as a reference. If a molecule ionizes from an almost spherical orbital like $\mathrm{D}_{2}$, then $\phi_{\mathrm{HHG}}$ is $\sim \theta_{c}$. Since $\theta_{c}$ is a characteristic of the continuum electron and not the unaligned molecule, $\theta_{c}$ does not change for different molecules for the same phase delay and the same laser intensities. Then we compare $\phi_{\mathrm{HHG}}$ for $\mathrm{D}_{2}$ with $\mathrm{N}_{2}$ and $\mathrm{CO}_{2}$ at given delay and harmonic number to identify the symmetry.

To produce the $400 \mathrm{~nm}$ laser pulse, we pass an $800 \mathrm{~nm}$, $1 \mathrm{kHz}, 35 \mathrm{fs}$, laser pulse generated from a Ti:sapphire laser system (KM Labs) through a $300 \mu \mathrm{m}$ thick BBO crystal. We compensate the group delay between the two laser pulses with a $0.65 \mathrm{~mm}$ thick calcite plate. Rotating the calcite plate accumulates an extra optical path, which delays one of the laser pulses with an accuracy of $<50$ as. We focus the collinear laser beams into a pulsed gas jet using a $50 \mathrm{~cm}$ focal length mirror. The generated high-harmonic emission is spectrally dispersed by an aberration-corrected concave grating and imaged onto a microchannel plate and recorded by a CCD camera. We integrate the images over the spatial dimension to obtain the high-harmonic spectrum. We estimate the 800 and $400 \mathrm{~nm}$ laser intensity to be $\sim 1.1 \times 10^{14} \mathrm{~W} / \mathrm{cm}^{2}$ and $\sim 0.8 \times 10^{14} \mathrm{~W} / \mathrm{cm}^{2}$, respectively, by utilizing the cutoff photon energy.

Figure 2 shows the high-harmonic spectra of (a) $\mathrm{D}_{2}$ and (b) $\mathrm{CO}_{2}$ as a function of the two-color delay. We record the spectra for $\mathrm{N}_{2}$ as well (see supplementary material [18]). Zero time delay in Fig. 2 is arbitrarily chosen. We do not need to know zero time delay accurately to identify the symmetry of a molecular orbital. As previously reported [15,19-22], even harmonics are observed as well as the odd harmonics. The spectral intensity of each harmonics is modulated with the period of $\sim 0.67 \mathrm{fs}$ - half of the optical period of the $400 \mathrm{~nm}$ light. This is due to the fact that the same magnitude of the laser field is repeated at every half optical period of the $400 \mathrm{~nm}$ laser pulse.

For $\mathrm{D}_{2}$, the peak of the high-harmonic emission is centered at the delay of $\sim 0.33 \mathrm{fs}$ ( and $\sim 1.00 \mathrm{fs}$ ) at the lowest harmonic number. The peak shifts first to smaller and then to larger phases as the harmonic number increases. The even harmonics becomes weaker for the higher harmonics. In contrast, for $\mathrm{CO}_{2}$ we observe a very different behavior in the harmonic intensity, especially, at the low harmonics.

The time shift between two adjacent harmonics includes information on the polarization angle. Harmonics have their origin in the interference between the recolliding electron and the molecular orbital (Fig. 1). The polarization of the emitted light arises from this interference. In Fig. 3, we plot the polarization angle, $\phi_{\mathrm{HHG}}$, of the harmonics for (a) $\mathrm{D}_{2}$, (b) $\mathrm{N}_{2}$, and (c) $\mathrm{CO}_{2}$ as a function of the delay and the harmonic number. In order to obtain $\phi_{\mathrm{HHG}}$, we use Eq. (1) and choose the harmonic pair of 13 th $+2 n$ and 14 th $+2 n$, where $n$ is $0-4$, respectively. We interpolate the data value along the harmonic number. The difference between $\mathrm{D}_{2}$ (and $\mathrm{N}_{2}$ ) with $\sigma_{g}$ symmetry and $\mathrm{CO}_{2}$ with $\pi_{g}$ symmetry is clearly seen. At a delay of $\sim 0.30 \mathrm{fs}$ and at harmonic $\sim 13, \phi_{\mathrm{HHG}}$ is $\sim 45^{\circ}$ for $\mathrm{D}_{2}$ while $\phi_{\mathrm{HHG}}$ is $\sim 0^{\circ}$ for $\mathrm{CO}_{2}$. On the other hand, at the delay of $\sim 0.67 \mathrm{fs}, \phi_{\mathrm{HHG}}$ is $\sim 0^{\circ}$ for $\mathrm{D}_{2}$, while $\phi_{\mathrm{HHG}}$ for $\mathrm{CO}_{2}$ is $\sim 90^{\circ}$.

In Fig. 3(d), we plot the result of a classical electron trajectory calculation for $\theta_{c}$ under the two-color laser fields. We use strong field approximation and assume a 

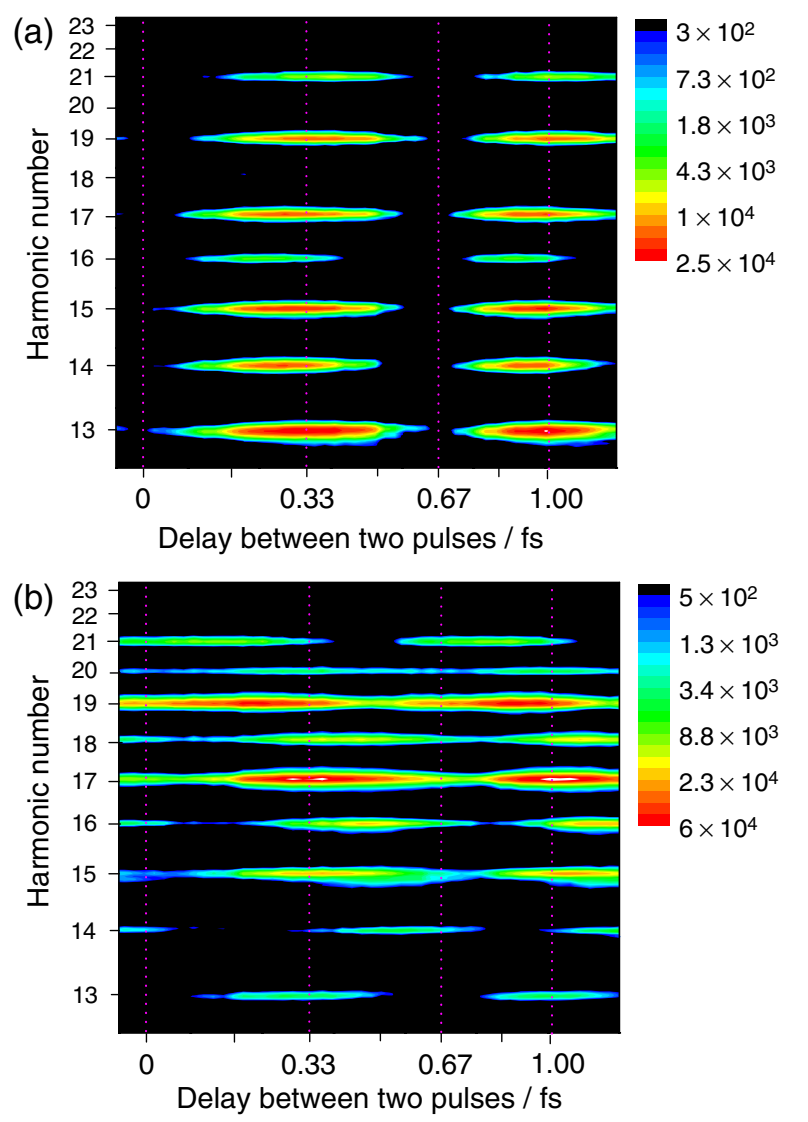

FIG. 2 (color online). High harmonic generation spectra of (a) $\mathrm{D}_{2}$ and (b) $\mathrm{CO}_{2}$ as a function of the phase delay between the two laser pulses. The color code represents the signal intensity.

totally spherical cross section. Figure 3(a) is comparable to Fig. 3(d) except for the sign of the angle which cannot be distinguished experimentally. This confirms that $\theta_{c}$ can be controlled by changing the phase delay, and that $\phi_{\mathrm{HHG}}$ for $\mathrm{D}_{2}$ is a measure of $\theta_{c}$. While the absolute value of $\theta_{c}$ depends on the intensity ratio between the $800 \mathrm{~nm}$ and the $400 \mathrm{~nm}$ laser pulses, the overall structure of the distribution remains the same.

The orbital symmetry dependence of the high-harmonic polarization, that is immediately obvious in Fig. 3, is shown differently in Fig. 3. The square data points represent $\phi_{\mathrm{HHG}}$ for (a) $\mathrm{N}_{2}$ and (b) $\mathrm{CO}_{2}$ versus $\phi_{\mathrm{HHG}}$ for $\mathrm{D}_{2}$, regarded as $\theta_{c}$, for the $\sim 13$ th harmonics. For $\mathrm{N}_{2}$, the slope is positive while for $\mathrm{CO}_{2}$ the slope is negative. To confirm that the slope is related to the symmetry of the molecular orbital, we simulate $\phi_{\mathrm{HHG}}$ as a function of $\theta_{c}$. We use the HOMO $\left(\pi_{g}\right)$, the HOMO-1 $\left(\pi_{u}\right)$, and the HOMO-2 $\left(\sigma_{g}\right)$ of $\mathrm{CO}_{2}$ calculated by the GAMESS program [23]. We calculate the magnitude of the dipole moment induced along the 800 $\mathrm{nm}(400 \mathrm{~nm})$ polarization axis as a function of $\theta_{c}$ for the harmonic number at 13th and 14th, respectively, and then obtain $\phi_{\mathrm{HHG}}$. We average $\phi_{\mathrm{HHG}}$ over the angular distribution of the molecular axis weighted by the ionization probability [10]. The details of the calculation are described in the supplementary material [18].

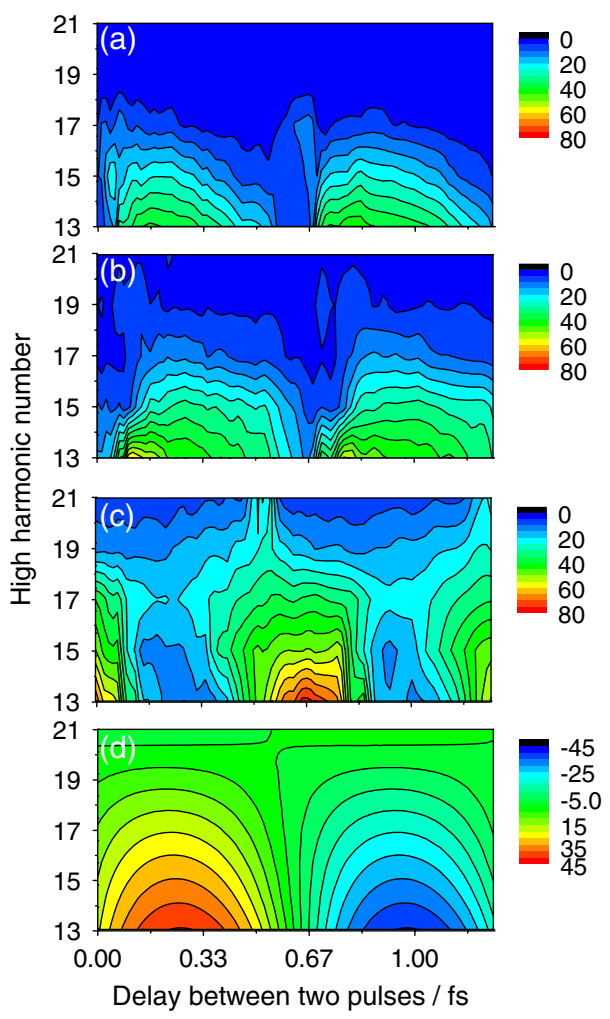

FIG. 3 (color online). Polarization angle, $\phi_{\mathrm{HHG}}$, as function of the delay and the harmonic number for (a) $\mathrm{D}_{2}$, (b) $\mathrm{N}_{2}$, and (c) $\mathrm{CO}_{2}$, respectively. The angle is represented by the color coding. In (d), we plot the calculated $\theta_{c}$ for a spherically symmetric orbital.

The dashed line in Fig. 4(a) represents $\phi_{\mathrm{HHG}}$ as a function of $\theta_{c}$ when the orbital is totally spherical. In this case, $\phi_{\mathrm{HHG}}$ is proportional to $\theta_{c}$. The observed data points for $\mathrm{N}_{2}$ are slightly larger than the dotted line at a given $\theta_{c}$. For $\mathrm{N}_{2}$, the tunnel ionization probability of the $2 p \quad \sigma_{g}$ orbital has an elliptical distribution along the molecular axis [14] and the orbital is elongated along the molecular axis. These facts result in the slightly large $\phi_{\mathrm{HHG}}$ at a given $\theta_{c}$.

In Fig. 4(b), we plot the calculated $\phi_{\mathrm{HHG}}$ versus $\theta_{c}$ for the $\pi_{g}$ orbital as a solid line and for the $\pi_{u}$ orbital as a dashed line. The calculated line for the $\pi_{g}$ has the same tendency as the observed data points, which is clearly different from the $\pi_{u}$. For the $\sigma_{g}$ case, the slope of $\phi_{\mathrm{HHG}}$ versus $\theta_{c}$ is positive as is the case of $\mathrm{N}_{2}$ 's $\sigma_{g}$ state. It is consistent that because the $\pi_{g}$ orbital has the most loosely bound state for $\mathrm{CO}_{2}$, as one might expect for unaligned molecules, the $\pi_{g}$ orbital contributes predominantly to the high-harmonic generation from $\mathrm{CO}_{2}$ at this laser intensity.

We have plotted $\phi_{\mathrm{HHG}}$ as a function of $\theta_{c}$ in a laboratory frame. This requires minimum assumptions yet gives a clear identification of the molecular orbital symmetry. For comparison with the alignment approach [16], we plot $\left|\phi_{\mathrm{HHG}}-\theta_{c}\right|$ versus $\theta_{c(\mathrm{MF})}$ in the online material, where $\theta_{c(\mathrm{MF})}$ is the recollision angle in the molecular 


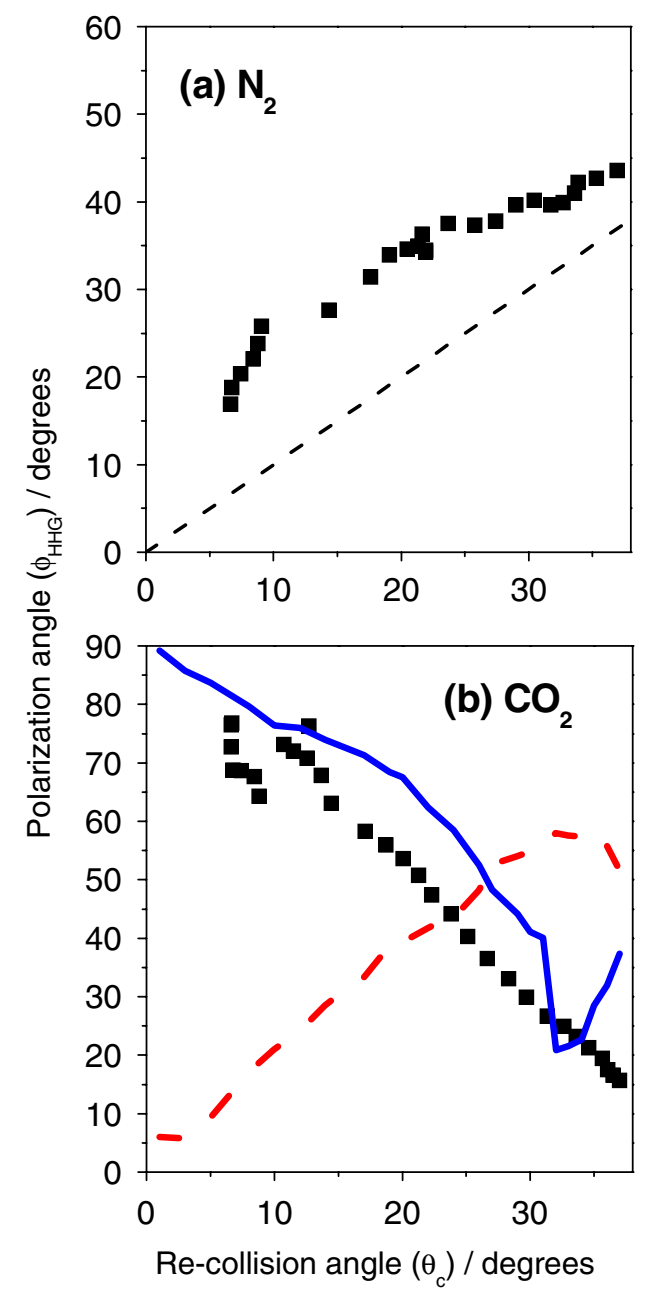

FIG. 4 (color online). The square data points show the measured $\phi_{\mathrm{HHG}}$ as a function of $\theta_{c}$ at the $\sim 13$ th harmonic for (a) $\mathrm{N}_{2}$, (b) $\mathrm{CO}_{2}$. The dotted line in (a) is a plot of the calculated result using a spherically symmetric molecular orbital. The solid and dashed lines in (b) are the plots of the calculated $\phi_{\mathrm{HHG}}$ for the $\pi_{g}$ and the $\pi_{u}$ orbital of $\mathrm{CO}_{2}$, respectively.

frame. In our results, it is possible to have a perpendicular polarization component when the electron recollides with the $\pi_{g}$ orbital parallel to the molecular axis. This is due to the fact that the continuum carries the phase of the bound state. On the other hand, the alignment approach results in $\left|\phi_{\mathrm{HHG}}-\theta_{c}\right| \sim 0$ at $\theta_{c(\mathrm{MF})} \sim 0$ in the molecular frame, i.e., for the molecular axis aligned parallel to the field, by symmetry the emission must be polarized parallel to the laser field.

In summary, we have identified the orbital symmetry of the $\pi_{g}$ orbital using high-harmonic spectroscopy, which was revealed despite the complexities and uncertainties. Our results lay out a general path to tomography of complex orbitals. We have shown that two-color fields will replace alignment in the role of obtaining multiple projections of an orbital. Combining the two-color fields, alignment will gain a new role- to select the orbital of interest.
Our approach can be extended to dynamical measurements. If, for example, a nonadiabatic process occurs at the time of tunnel ionization, then the molecular orbital may change its spatial distribution dynamically, which leads to dynamical changes in $\phi_{\mathrm{HHG}}$. Because high-harmonic order can be related to the recollision time [3], dynamical changes in the molecular orbital can be mapped onto a two-dimensional figure like Fig. 3. In Fig. 3 , the $\phi_{\text {HHG }}$ peak appears at a delay $\sim 0.30 \mathrm{fs}$ for the $\sigma_{g}$ orbital, while the $\phi_{\text {HHG }}$ peak appears at a delay $\sim 0.67$ fs for the $\pi_{g}$ orbital. If the molecular symmetry changes from $\sigma_{g}$ to $\pi_{g}$, then the changes can be traced on the map.

For our experimental conditions, the $\sigma_{g}$ and $\pi_{u}$ orbitals cannot be distinguished because the $\theta_{c}$ range is limited from $0^{\circ}$ to $\sim 45^{\circ}$. If we control the electron trajectory using a longer laser wavelength, for instance, combining 800 with $1600 \mathrm{~nm}$ laser pulses, we can extend the angles $\theta_{c}$ to $\sim 90^{\circ}$. In addition, a longer laser wavelength can extend the cutoff harmonics, permitting us to measure threedimensional molecular structure for any orbital symmetry and view dynamical changes with attosecond time resolution.

It is our pleasant duty to acknowledge extensive advice and assistance from Dr. A. Naumov. We acknowledge funding from JST and NSERC.

[1] S. Baker et al., Phys. Rev. Lett. 101, 053901 (2008).

[2] W. Li et al., Science 322, 1207 (2008).

[3] H. Niikura, D. M. Villeneuve, and P. B. Corkum, Phys. Rev. Lett. 94, 083003 (2005).

[4] J. Itatani et al., Nature (London) 432, 867 (2004).

[5] H. J. Wrner et al., Phys. Rev. Lett. 102, 103901 (2009).

[6] T. Morishita, A. T. Le, Z. Chen, and C. D. Lin, Phys. Rev. Lett. 100, 013903 (2008).

[7] M. Kitzler et al., New J. Phys. 10, 025029 (2008).

[8] P. Corkum, Phys. Rev. Lett. 71, 1994 (1993).

[9] F. Krausz and M. Ivanov, Rev. Mod. Phys. 81, 163 (2009).

[10] D. Pavicic et al., Phys. Rev. Lett. 98, 243001 (2007).

[11] C. D. Lin, X. M. Tong, and Z. X. Zhao, J. Mod. Opt. 53, 21 (2006).

[12] O. Smirnova et al., Phys. Rev. Lett. 102, 063601 (2009).

[13] O. Smirnova et al., Nature (London) 460, 972 (2009).

[14] Y. Mairesse et al., New J. Phys. 10, 025015 (2008).

[15] D. Shafir et al., Nature Phys. 5, 412 (2009).

[16] J. Levesque et al., Phys. Rev. Lett. 99, 243001 (2007).

[17] T. Zuo, A. D. Bandrauk, M. Ivanov and P. B. Corkum, Phys. Rev. A 51, 3991 (1995).

[18] See supplementary material at http://link.aps.org/ supplemental/10.1103/PhysRevLett.105.053003 for the detailed description of the analysis.

[19] N. Dudovich et al., Nature Phys. 2, 781 (2006).

[20] T. T. Liu, T. Kanai, T. Sekikawa, and S. Watanabe, Phys. Rev. A 73, 063823 (2006).

[21] I. J. Kim et al., Phys. Rev. Lett. 94, 243901 (2005).

[22] S. Gilbertson et al., Appl. Phys. Lett. 92, 071109 (2008).

[23] M. W. Schmidt et al., J. Comput. Chem. 14, 1347 (1993). 